\title{
A Favorable Response to Cisplatin, Pemetrexed and Bevacizumab in Two Cases of Invasive Mucinous Adenocarcinoma Formerly Known as Pneumonic-type Mucinous Bronchioloalveolar Carcinoma
}

\author{
Hideaki Yamakawa ${ }^{1,3}$, Noboru Takayanagi ${ }^{1}$, Takashi Ishiguro ${ }^{1}$, \\ Naho Kagiyama ${ }^{1}$, Yoshihiko Shimizu ${ }^{2}$ and Yutaka Sugita ${ }^{1}$
}

\begin{abstract}
The leading subtype of lung cancer, adenocarcinoma, received a new classification in 2011 when multifocal, pneumonic-type mucinous tumors, formerly called mucinous bronchioloalveolar carcinomas, were reclassified as "invasive mucinous adenocarcinomas." These tumors appear to be less sensitive to chemotherapy than other non-small cell lung cancers. We herein report two cases of invasive mucinous adenocarcinoma that showed a dramatic radiologic response to combination therapy with cisplatin, pemetrexed and bevacizumab. Recent data suggest that the use of paclitaxel-based chemotherapy is an acceptable therapeutic strategy in cases of invasive mucinous adenocarcinoma. The cases reported here and preclinical findings suggest the therapeutic efficacy of cisplatin, pemetrexed and bevacizumab in treating such tumors.
\end{abstract}

Key words: invasive mucinous adenocarcinoma, pemetrexed, bevacizumab, chemotherapy, mucinous bronchioloalveolar carcinoma

(Intern Med 52: 2781-2784, 2013)

(DOI: 10.2169/internalmedicine.52.0766)

\section{Introduction}

In 2011, a new classification of adenocarcinoma of the lungs was published $(1,2)$. One of the major changes is the reclassification of the carcinoma formerly known as mucinous bronchioloalveolar carcinoma (BAC) to "invasive mucinous adenocarcinoma (IMA)." IMA was placed in a new category due to its unique radiologic, morphologic and genetic characteristics. There are reports of greater chemoresistance in tumors previously categorized as BAC $(3,4)$. In a prospective study comparing four platinum-based chemotherapy regimens as first-line therapy in advanced non-small cell lung cancer (NSCLC) patients, the Eastern Cooperative Oncology Group (ECOG) 1594 trial, the response rate of advanced BAC to chemotherapy was lower than that of other NSCLC pathological subtypes [6\% versus 20\% (5)]. We experienced two cases of IMA that showed a rapid and dramatic response to combination therapy with cisplatin, pemetrexed and bevacizumab. We herein report on these cases with a special focus on the efficacy of this treatment regimen.

\section{Case Reports}

\section{Case 1}

A 65-year-old man, a current smoker (Brinkman index: 1410), presented with a productive cough with sputum and dyspnea on exertion; however, he had maintained an ECOG Performance Status (PS) of 1. Auscultation of the lungs revealed bilateral coarse and fine crackles. Chest computed tomography (CT) showed diffuse pulmonary parenchymal involvement with bilateral ground-glass opacity and consolidation with emphysema, predominantly in the lower lobes (Fig. 1A, B). The patient was diagnosed via a transbronchial

${ }^{1}$ Department of Respiratory Medicine, Saitama Cardiovascular and Respiratory Center, Japan, ${ }^{2}$ Department of Diagnostic Pathology, Saitama Cardiovascular and Respiratory Center, Japan and ${ }^{3}$ Department of Respiratory Medicine, Jikei University School of Medicine, Japan Received for publication April 12, 2013; Accepted for publication July 17, 2013

Correspondence to Dr. Hideaki Yamakawa, hide1144@jikei.ac.jp 

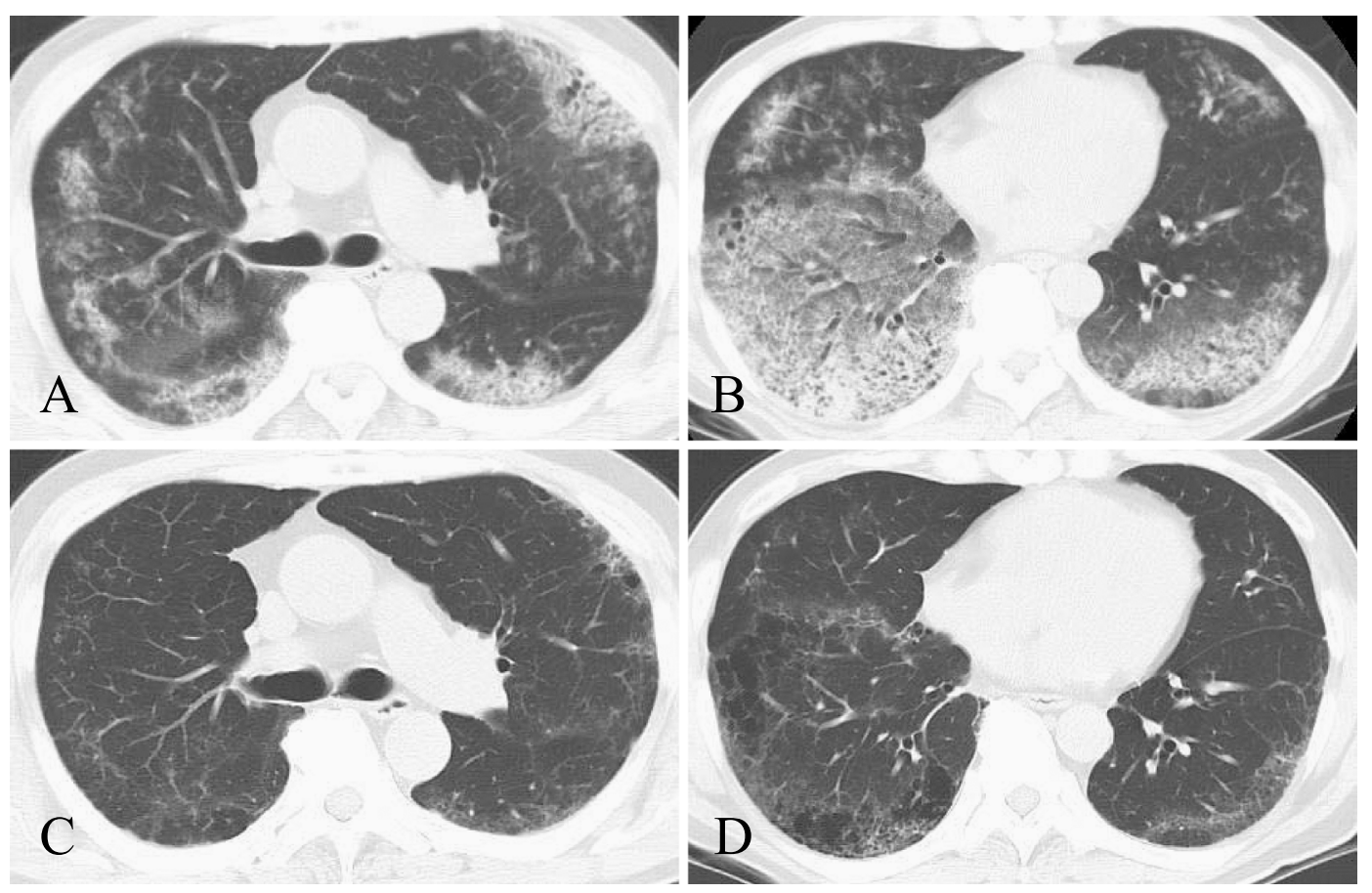

Figure 1. Case 1: Chest computed tomography performed on initial presentation (upper panels) and after four cycles of chemotherapy (lower panels). A, B: Diffuse pulmonary parenchymal involvement with bilateral ground-glass opacity and consolidation with emphysema, predominantly in the lower lobes. C, D: The radiological response was considered to be nearly complete after four cycles of chemotherapy.

biopsy in June 2012 as having IMA with negative epidermal growth factor receptor (EGFR) polysomy/mutations and the echinoderm microtubule-associated protein-like 4 (EML 4)ALK fusion gene. CT, brain magnetic resonance imaging (MRI) and bone scintigraphy revealed no evidence of extrathoracic metastasis. The clinical staging was stage IV [cT4N0M1a (PUL)]. An immunohistochemical analysis was positive for cytokeratin (CK) 7 and negative for CK20 and thyroid transcription factor-1 (TTF-1).

The patient underwent first-line treatment with cisplatin $\left(75 \mathrm{mg} / \mathrm{m}^{2}\right)$, pemetrexed $\left(500 \mathrm{mg} / \mathrm{m}^{2}\right)$ and bevacizumab $(15$ $\mathrm{mg} / \mathrm{kg}$ ) starting on day 1 every 21-28-day cycle, along with folic acid and vitamin B12 supplementation. The radiologic response was considered to be nearly complete after four cycles (Fig. 1C, D). No treatment-related toxicities were apparent. Following the completion of these treatments, pemetrexed and bevacizumab were continued as maintenance therapy every 21 days. The patient suffered a clinical deterioration that led to a cough with sputum again as a result of disease progression after three cycles of maintenance therapy.

\section{Case 2}

A 73-year-old woman, a current smoker (Brinkman index: 840 ), presented with a productive cough with sputum of five months duration, although she had maintained an ECOG PS of 0 . Auscultation of the lungs revealed bilateral coarse and fine crackles. CT showed bilateral pulmonary alveolar consolidation with ground-glass opacity and emphysema, par- ticularly in the left upper and lower lobes and right lower lobe (Fig. 2A, B). The patient was diagnosed via a transbronchial biopsy in August 2012 as having IMA with negative EGFR polysomy/mutations and the EML 4-ALK fusion gene. There was no evidence of extrathoracic metastasis on abdominal CT, brain MRI or bone scintigraphy. The clinical staging was stage IV [cT4NOM1a (PUL)]. An immunohistochemical analysis was positive for CK7 and negative for CK20 and TTF-1.

The patient underwent first-line treatment with cisplatin $\left(75 \mathrm{mg} / \mathrm{m}^{2}\right)$, pemetrexed $\left(500 \mathrm{mg} / \mathrm{m}^{2}\right)$ and bevacizumab $(15$ $\mathrm{mg} / \mathrm{kg}$ ) starting on day 1 every 28 days, along with folic acid and vitamin B12 supplementation. After one cycle, mild renal failure appeared, and the dose of cisplatin was reduced to $75 \%$ of the initial dose for the following cycles. A major radiologic response was achieved after four cycles (Fig. 2C, D). The only treatment-related toxicity was mild renal failure. Following the completion of these treatments, pemetrexed and bevacizumab were continued as maintenance therapy every 28 days. After four cycles of maintenance therapy, the patient did not present with any clinical symptoms; however, she a exhibit a radiological deterioration.

\section{Discussion}

IMA, formerly known as mucinous BAC, is derived from metaplasia of the bronchiolar epithelia. It presents more frequently as a pneumonic-type infiltrate, rarely demonstrates 


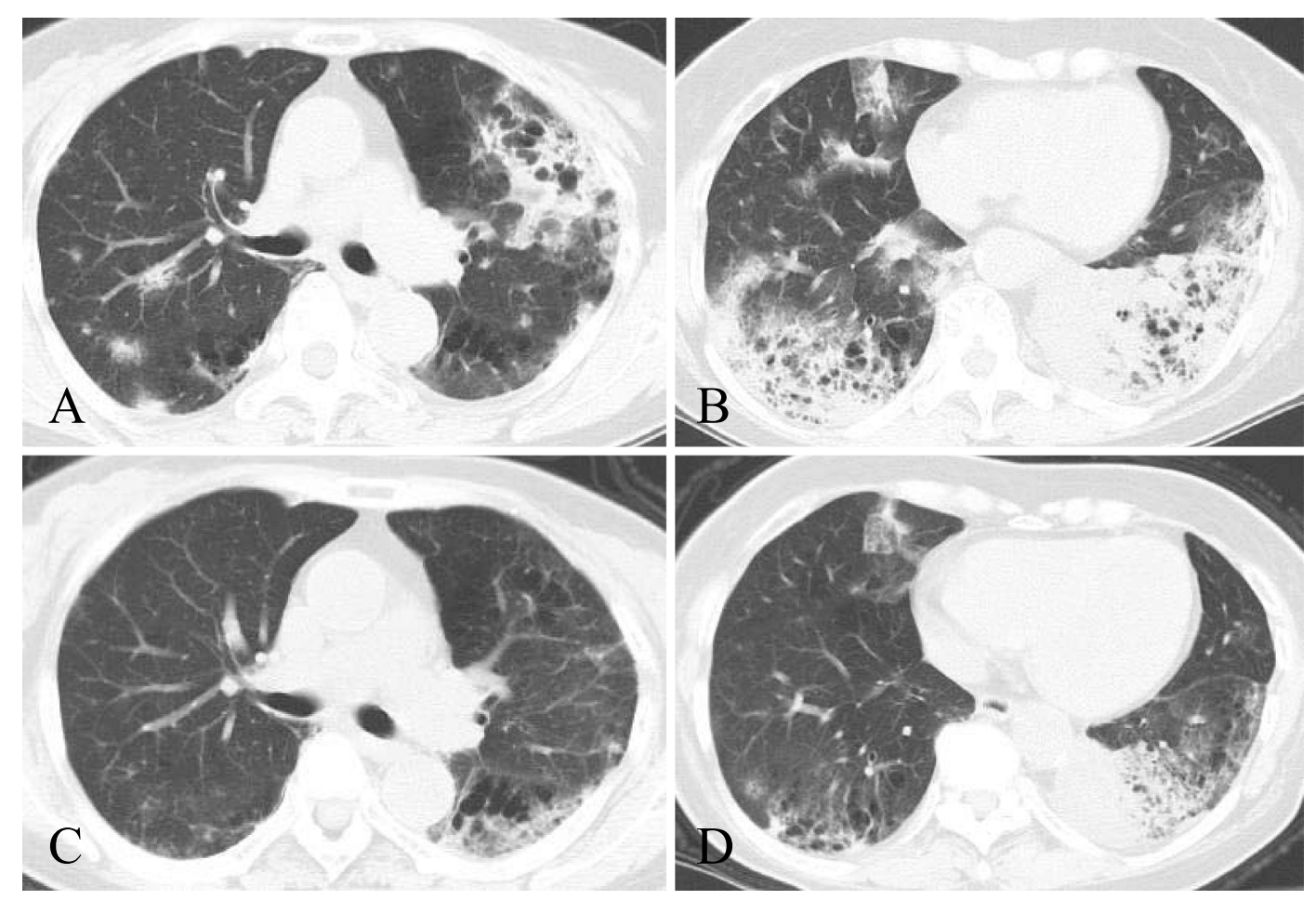

Figure 2. Case 2: Chest computed tomography performed on initial presentation (upper panels) and after four cycles of chemotherapy (lower panels). A, B: Bilateral pulmonary alveolar consolidation with ground-glass opacity and emphysema, particularly in the left upper and lower lobes and right lower lobe. C, D: A major radiological response was evident after four cycles of chemotherapy.

EGFR polysomy/mutations and much more frequently harbors and is driven by K-ras mutations (6). On immunohistochemistry, the mucinous type is positive for CK7; however, in contrast to the nonmucinous type, the mucinous type is also typically positive for CK20 and negative for TTF$1(7,8)$. Recently, immunohistochemistry studies of the mucinous type have given variable results. TTF-1, if positive, is weak and patchy, while CK7 is usually somewhat positive, with CK20 being even less so (6). These oncogenic cytological characteristic differences may lead to different therapeutic responses.

We experienced two cases in which cisplatin, pemetrexed and bevacizumab were effective for IMA. The AVAPERL study (MO22089), presented at the 2011 European Multidisciplinary Cancer Congress, revealed significant efficacy and safety of this therapeutic regimen against advanced NSCLC (9); however, the frequency of IMA in that study was not evident. Historically, mucinous-type BAC has appeared to be resistant to EGFR tyrosine kinase inhibitors (EGFR-TKIs) and may be more responsive to taxane-based chemotherapy; the objective response rate of paclitaxel following a 96-hour infusion was reported to be $19 \%$ (10). The details of the cases reported here suggest the therapeutic efficacy of combination therapy with cisplatin, pemetrexed and bevacizumab in patients with IMA.

There are only two reports of IMA exhibiting a durable response to pemetrexed $(11,12)$. Pemetrexed, a potent antifolate recently developed as an effective agent for NSCLC, targets three enzymes involved in nucleic acid synthesis, in particular thymidylate synthase (TS) (13). Takezawa et al. reported that a high level of the TS expression in NSCLC tumors is significantly associated with a reduced tumor response and a shorter progression-free survival in patients treated with pemetrexed combined with platinum agents. They suggest that the TS expression is a potential predictive marker for chemosensitivity to pemetrexed in patients with NSCLC (14). Moreover, it has been suggested that a lower TS expression in adenocarcinoma of the lungs may explain the higher antitumor activity of TS-inhibiting agents, such as pemetrexed (15). With a low level of expression being favorable in terms of a response, the expression of TS may be low in IMA patients. However, the TS gene expression in tumors diagnosed as IMA and other types of adenocarcinoma has not been reported.

In contrast, bevacizumab, a monoclonal antibody active against vascular endothelial growth factor (VEGF), has shown efficacy in patients with a variety of cancers. The addition of bevacizumab to a standard, platinum-based chemotherapy regimen improves overall survival in patients with advanced non-squamous cell NSCLC and a good ECOG PS. In addition, bevacizumab prolongs progression-free survival and improves the response rate (16). Jain reported that an elevated VEGF level causes a disorganized and "leaky" vasculature within the tumor; this effect elevates interstitial pressure and thus decreases the delivery of chemotherapeutic agents to the tumor (17). Subsequently, Willett et al. found that bevacizumab increases drug delivery to the tumor (18). This mechanism may explain the response to chemotherapy 
observed in our cases.

Our two cases suggest a high sensitivity of IMA to combination therapy with cisplatin, pemetrexed and bevacizumab. Further prospective studies are needed to clarify the efficacy of this therapeutic regimen for the management of IMA.

The authors state that they have no Conflict of Interest (COI).

\section{References}

1. Travis WD, Brambilla E, Noguchi M, et al. International Association for the Study of Lung Cancer/American Thoracic Society/ European Respiratory Society international multidisciplinary classification of lung adenocarcinoma. J Thorac Oncol 6: 244-285, 2011.

2. Pelosi G. The new taxonomy of lung adenocarcinoma stemming from a multidisciplinary integrated approach: novel pathology concepts and perspectives. J Thorac Oncol 6: 241-243, 2011.

3. Delarue NC, Anderson W, Sanders D, et al. Bronchiolo-alveolar carcinoma. A reappraisal after 24 years. Cancer 29: 90-97, 1972.

4. Edgerton F, Rao U, Takita H, et al. Bronchio-alveolar carcinoma. A clinical overview and bibliography. Oncology 38: 269-273, 1981.

5. Kris MG, Giaccone G, Davies A, et al. Systemic therapy of bronchioloalveolar carcinoma: results of the first IASLC/ASCO consensus conference on bronchioloalveolar carcinoma. J Thorac Oncol 1 (9 Suppl): S32-S36, 2006

6. Garfield DH, Cadranel J, West HL. Bronchioloalveolar carcinoma: the case for two diseases. Clin Lung Cancer 9: 24-29, 2008.

7. Goldstein NS, Thomas M. Mucinous and nonmucinous bronchioloalveolar adenocarcinomas have distinct staging patterns with thyroid transcription factor and cytokeratin 20 antibodies. Am J Clin Pathol 116: 319-325, 2001.

8. Shah RN, Badve S, Papreddy K, et al. Expression of cytokeratin 20 in mucinous bronchioloalveolar carcinoma. Hum Pathol 33:
915-920, 2002

9. Barlesi F, de Castro J, Dvornichenko V, et al. AVAPERAL (MO 22089): final efficacy outcomes for patients (pts) with advanced non-squamous non-small cell lung cancer (nsNSCLC) randomised to continuation maintenance (mtc) with bevacizumab (bev) or bev+pemetrexed (pem) after first-line (1L) bev-cisplatin (cis)-pem treatment (Tx). Paper presented at: 2011 European Multidisciplinary Cancer Congress; September 23-27, 2011; Stockholm, Sweden.

10. West HL, Crowley JJ, Vance RB, et al. Advanced bronchioloalveolar carcinoma: a phase II trial of paclitaxel by 96-hour infusion (SWOG 9714): a Southwest Oncology Group Study. Ann Oncol 16: 1076-1080, 2005.

11. Manson GV, Ma PC. Response to pemetrexed chemotherapy in lung adenocarcinoma-bronchioloalveolar carcinoma insensitive to erlotinib. Clin Lung Cancer 11: 57-60, 2010.

12. Garfield DH, Franklin W. Dramatic response to pemetrexed in a patient with pneumonic-type mucinous bronchioloalveolar carcinoma. J Thorac Oncol 6: 397-398, 2011.

13. Chattopadhyay S, Moran RG, Goldmann ID. Pemetrexed: biochemical and cellular pharmacology, mechanisms, and clinical applications. Mol Cancer Ther 6: 404-417, 2007.

14. Takezawa K, Okamoto I, Okamoto W, et al. Thymidylate synthase as a determinant of pemetrexed sensitivity in non-small cell lung cancer. Br J Cancer 104: 1594-1601, 2011.

15. Tanaka F, Wada H, Fukui Y, et al. Thymidylate synthase (TS) gene expression in primary lung cancer patients: a large-scale study in Japanese population. Ann Oncol 22: 1791-1797, 2011.

16. Sandler A, Gray R, Perry MC, et al. Paclitaxel-carboplatin alone or with bevacizumab for non-small-cell lung cancer. N Engl $\mathrm{J}$ Med 355: 2542-2550, 2006.

17. Jain RK. Normalizing tumor vasculature with anti-angiogenic therapy: a new paradigm for combination therapy. Nat Med 7: 987989, 2001.

18. Willett CG, Boucher Y, di Tomaso E, et al. Direct evidence that the VEGF-specific antibody bevacizumab has antivascular effects in human rectal cancer. Nat Med 10: 145-147, 2004.

(C) 2013 The Japanese Society of Internal Medicine http://www.naika.or.jp/imonline/index.html 